\title{
In Vitro Fertilization Pregnancy Outcomes and Effect of Atosiban Among Women With Endometriosis in Presence or Absence of Adenomyosis
}

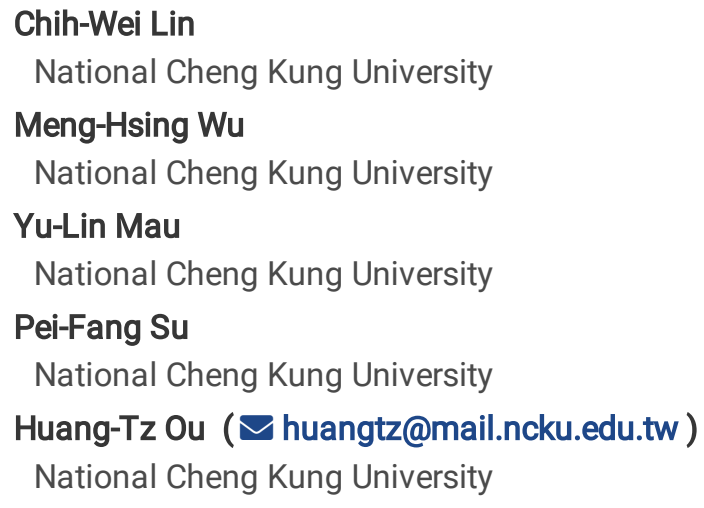

\section{Research Article}

Keywords: adenomyosis, endometriosis, in vitro fertilization, pregnancy, atosiban

Posted Date: October 13th, 2021

DOI: https://doi.org/10.21203/rs.3.rs-963721/v1

License: (c) (i) This work is licensed under a Creative Commons Attribution 4.0 International License. Read Full License 


\section{Abstract}

Background

The use of atosiban during embryo transfer (ET), an oxytocin receptor antagonist, has been demonstrated to enhance pregnancy rate among infertile patients with endometriosis. However, its efficacy has not been assessed among those with concurrent adenomyosis, which may further affect pregnancy rate.

Methods

This retrospective cohort study assessed in vitro fertilization (IVF) pregnancy, and the effect of atosiban among 34 women with both endometriosis and adenomyosis (with 66 ET cycles), compared to 34 endometriosis patients without adenomyosis (with 54 ETs) and 38 patients with tubal factor (with $56 \mathrm{ETs}$ ). Multivariable logistic generalized estimating equation analyses were performed to assess pregnancy outcomes with adjustment for maternal and treatment characteristics.

Results

Significantly higher chances of biochemical pregnancy and live birth among endometriosis patients without adenomyosis versus those with both endometriosis and adenomyosis were found (odds ratios [95\% confidence intervals]: 2.981 [1.307, 6.803]; $p=0.009$, 2.694 [1.151, 6.304]; $p=0.022$. A significant positive association between atosiban use and biochemical pregnancy existed among endometriosis cases without adenomyosis (a 2.43-fold [1.01, 5.89] increase in successful pregnancy; $p<0.05$ ), but not for the other groups. An insignificant increase in pregnancy rates was found for atosiban-treated extensive adenomyosis cases (i.e., $\geq 50 \%$ ) versus non-treated cases (biochemical pregnancy, ongoing pregnancy, and live birth: $42.9 \%$ versus $33.3 \%, 37.5 \%$ versus $14.3 \%$, and $25.0 \%$ versus $14.3 \%$, respectively).

Conclusions

Poor pregnancy outcomes among adenomyosis-affected women were confirmed. The use of atosiban significantly enhanced IVF pregnancy among endometriosis patients without adenomyosis. Atosiban use might benefit those with more extensive adenomyosis.

\section{Introduction}

Endometriosis-related infertility is well-recognized. The presence of adenomyosis could further worsen the pregnancy outcomes of women with endometriosis. Endometrial-myometrial interface disruption has recently been hypothesized for the pathogenesis of adenomyosis [1]. Uterine hyperperistalsis may induce this disruption, by which injury leads to tissue hypoxia that incorporates epithelial-mesenchymal transition and bone-marrow-derived stem cell recruitment, and subsequently results in the formation of adenomyotic lesions in the myometrium. Furthermore, various dysregulations associated with gene expression (e.g., the disruption of decidualization and cell adhesion) that could negatively affect endometrial receptivity and embryo implantation have been reported [2].

In vitro fertilization (IVF) is a promising treatment for infertility caused by various factors (e.g., endometriosis, tubal factor). Several strategies have been developed for enhancing the pregnancy rate of IVF. Considering the adverse effects of frequent uterine contractions following embryo transfer (ET) in IVF treatment, treatments that inhibit uterine contractions have been proposed [3]. Among them, atosiban (Tractocile ${ }^{\circledR}$ ), a mixture of vasopressin V1 and an oxytocin receptor antagonist that can lower uterine contraction frequency and possibly enhance endometrial perfusion and endometrial receptivity, has been shown to improve the pregnancy rate following ET [4]. Although evidence on the effects of atosiban use on the pregnancy outcomes of general infertility patients undergoing IVF remains inconclusive $[5,6,7]$, the efficacy of atosiban on clinical pregnancy and implantation rates following ET among women with endometriosis has been confirmed by a randomized controlled trial [8]. However, the effect of atosiban among patients with both endometriosis and adenomyosis has not yet been investigated. The effect of atosiban on the IVF pregnancies for endometriosis patients with different clinical presentations of adenomyosis remains uncertain.

This study therefore assessed the pregnancy outcomes among women with endometriosis undergoing IVF treatment stratified by the presence or absence of adenomyosis and its subtypes. The effectiveness of atosiban for these populations was further investigated. 


\section{Materials And Methods \\ Study patients}

Infertile women undergoing IVF at the Assisted Reproductive Technology Center of National Cheng Kung University Hospital (NCKUH), Tainan, Taiwan, between January 2007 and July 2019 were included in this study. The infertility treatment pertaining to the current study was carried out in accordance with relevant guidelines and regulations.

Demographics (e.g., age), laboratory measurements (e.g., body mass index [BMI], anti-Müllerian hormone [AMH], infertility cause [i.e., endometriosis, tubal factor]), and pregnancy information (e.g., gravidity, parity, number of ET failures, number of embryos transferred per ET cycle, day of ET, use of atosiban, pregnancy results) were obtained from electronic medical records and medical charts at NCKUH. Three study groups were specified: women with endometriosis but without adenomyosis (endometriosis-only group), those with both endometriosis and adenomyosis (endometriosis + adenomyosis group), and those with tubal factor only (tubal-only group).

\section{Anatomy and assessment for sub-features of adenomyosis}

All patients had undergone transvaginal ultrasound for measurement of uterine dimensions, including length, anteroposterior diameter, endometrial thickness, myometrial thickness, and uterine flexion. All adnexal and uterine lesions were measured and their sizes, echogenicity, contour, and position were recorded. For the assessment and classification of adenomyosis, the reporting guidelines proposed by Van den Bosch et al. [9] were adopted in this study. The sonographic features, including the location, differentiation, uterine layer involvement, and extent of adenomyosis, were assessed accordingly. Specifically, the location was assigned as the anterior or posterior wall, depending on where adenomyosis was found. Differentiation was classified into focal, diffuse, or mixed type based on the proportion of adenomyosis being surrounded by normal myometrium. With the junctional zone of the myometrium and serosa used as a reference, the uterine layer involved was classified into the inner, middle, or outer layer. The extent of involvement was classified as less than $25 \%, 25 \%$ to $50 \%$, or more than $50 \%$. The adenomyosis cases were stratified based on these ultrasound features (i.e., differentiation [focal or diffuse], layer [inner, middle or outer], location [anterior or posterior wall], and extent $[<25 \%, 25-50 \%,>50 \%])$.

\section{Treatment}

For each IVF cycle, controlled ovarian hyperstimulation was applied using either a gonadotropin-releasing hormone (GnRH) agonist or a $\mathrm{GnRH}$ antagonist protocol. Recombinant human chorionic gonadotropin was administered if leading follicles reached $18-20 \mathrm{~mm}$. Transvaginal oocyte retrieval was performed 34-36 hours later, followed by in vitro embryo culture with or without intracytoplasmic sperm injection. ET was performed with good-quality embryos on Day 2-6. The luteal phase was supported by progesterone supplement. For frozen ET cycles, good-quality embryos cryopreserved from previous IVF cycles were thawed and transferred.

For atosiban administration, a single bolus dose $(6.75 \mathrm{mg} / 0.9 \mathrm{~mL})$ was infused intravenously for more than 1 minute before ET, and the remaining dose $(30.75 \mathrm{mg} / 4.1 \mathrm{~mL})$ was diluted to $500 \mathrm{~mL}$ using normal saline and infused for 1.5 hours following the ET procedure.

\section{Pregnancy outcome measurements}

Pregnancy outcomes of interest included 1) biochemical pregnancy, which was confirmed by a $\beta$-human chorionic gonadotropin level of $>30 \mathrm{IU} / \mathrm{L}$ measured 14 days following ET, 2) ongoing pregnancy, which was confirmed by the appearance of a gestational sac with a viable fetal heartbeat at the $10^{\text {th }}$ week of gestational age, and 3 ) live birth, which was confirmed when a live fetus (or feti) was present at the $24^{\text {th }}$ week.

\section{Statistical analysis}


The descriptive statistics (i.e., mean, standard deviation, frequency, and proportion) of demographic characteristics and laboratory data of all study patients were tabulated and further stratified by the patient subgroup. The logistic generalized estimating equation (GEE) model was adopted to analyze the pregnancy outcomes of each study subgroup and the effect of atosiban, with adjustment for patient clinical characteristics that were significantly associated with pregnancy outcomes. The results of the GEE model are presented in terms of the odds ratio $(\mathrm{OR})$ and $95 \%$ confidence interval $(\mathrm{Cl})$. The pregnancy rates for atosiban-treated and non-treated cases in adenomyosis subgroups stratified by clinical ultrasound presentation (in terms of differentiation, layer, location, and extent of adenomyosis) were also estimated. The difference in pregnancy rates between atosiban users and non-users was further tested using Fisher's exact tests. Statistical tests were two-sided, with a $p$-value of less than 0.05 considered as significant.

\section{Results}

103 infertile women with a total of 176 ET cycles were included in analysis, where 34 (54), 34 (66) and 38 (56) patients (ET cycles) were in the endometriosis-only, endometriosis + adenomyosis, and tubal-only groups, respectively (Table 1). There was no significant difference in patient clinical and treatment characteristics between the endometriosis-only and endometriosis + adenomyosis groups and between the tubal-only and endometriosis + adenomyosis groups. The crude pregnancy rates for the endometriosis-only group were significantly higher than those for the endometriosis + adenomyosis group (i.e., biochemical pregnancy: $50.0 \%$ versus $41.1 \%$, $p=0.041$; live birth: $35.2 \%$ versus $25.9 \%, p=0.038$ ).

The GEE analysis results for the between-group pregnancy comparison are presented in Table 2. The endometriosis-only group had significantly higher successful pregnancy following ET compared to those of the endometriosis + adenomyosis group in terms of biochemical pregnancy (OR [95\% Cl]: 2.981 [1.307, 6.803], $p=0.009)$ and live birth $(2.694$ [1.151, 6.304], $p=0.022)$, but no significant difference in pregnancy outcomes was found between the tubal-only and endometriosis + adenomyosis groups.

The results on the effect of atosiban on IVF pregnancy outcomes are summarized in Fig. 1. A significant and positive association between atosiban use and biochemical pregnancy was identified for the endometriosis-only group (i.e., the use of atosiban was associated with a 2.43 -fold $[1.01,5.89]$ increase in successful pregnancy; $p<0.05)$, but not for the other patient subgroups. Furthermore, as shown in Table 3, which lists results stratified by the clinical ultrasound presentation of adenomyosis, an insignificant increase in pregnancy rates was found for extensive adenomyosis cases (i.e., $\geq 50 \%$ ) treated with atosiban versus nontreated cases (i.e., biochemical pregnancy, ongoing pregnancy and live birth: $42.9 \%$ versus $33.3 \%, 37.5 \%$ versus $14.3 \%$, and $25.0 \%$ versus $14.3 \%$, respectively).

\section{Discussion}

This is the first study to assess the pregnancy outcomes and the effect of atosiban use among women with endometriosis stratified by the presence or absence of adenomyosis and its ultrasound features. A comparison to patients with tubal factor only was also made. The results show significantly poorer pregnancy outcomes after IV treatment for the endometriosis + adenomyosis group compared to those for the endometriosis-only group. The administration of atosiban was associated with significantly increased successful pregnancies for the endometriosis-only group. Endometriosis patients with more extensive adenomyosis might also benefit from atosiban use.

\section{Poor IVF pregnancies among endometriosis patients with coexisting adenomyosis}

Previous studies have shown lower clinical pregnancy rates after IVF treatment among patients with adenomyosis compared to those without adenomyosis [10]. This lower pregnancy rate may depend on the severity of adenomyosis (defined based on the appearance of ultrasonic features); more severe and extensive adenomyosis was linked to poorer pregnancy outcomes [10]. Consistent with these previous studies, in this study, compared to endometriosis-only patients, those with adenomyosis had significantly lower successful biochemical pregnancy and live birth. The pathogenesis and pathophysiology for adenomyosisassociated infertility have been suggested. Enhanced by local hyperestrogenism, the effect of adenomyosis on the myometrial architecture and peristalsis can disrupt the transport of sperm [2]. In addition, endometrial receptivity could be compromised because of impaired decidualization attributable to adenomyosis [2]. Several possible factors that can exacerbate reproductive functions (e.g., 
molecular markers related to inflammation and cell adhesion) have been suggested for adenomyosis-affected individuals [2]. The inadequate decidualization due to adenomyosis generally makes embryo implantation more difficult in clinical practice [11].

\section{Effect of atosiban on IVF pregnancy among endometriosis cases with or without adenomyosis}

Improved pregnancy outcomes associated with the use of atosiban following ET have been found among infertile women with failed ET (i.e., repeated implantation failure) $[5,6,12,13,14)$ and endometriosis [8]. Of note, increased successful implantation and pregnancies following atosiban administration during frozen ET among women with endometriosis are supported by a randomized trial, in which frequent uterine contractions and the serum level of oxytocin were reduced [8]. However, it should be noted that those coexisting with adenomyosis were excluded from this trial. Substantial evidence on the pathologic dysregulation of the endometrial environment due to adenomyosis and its detrimental impact on fertility has implied that atosiban is a promising treatment for adenomyosis cases [2]. The present study thus analyzed endometriosis patients with adenomyosis. However, the overall adenomyosis patients (without stratified by the ultrasound features) did not appear to benefit from atosiban use in terms of IVF pregnancies.

This result may have been affected by the fact that all the patients with adenomyosis in our study were pre-treated with $\mathrm{GnRH}$ agonists before ET, which has been shown to increase clinical, implantation and ongoing pregnancy rates [15]. The use of atosiban after the administration of $\mathrm{GnRH}$ agonists might thus be ineffective. Future research is needed to understand the comparative reproductive efficacy of GnRH agonists against atosiban, and determine which one is more effective for successful IVF pregnancies. The clinical effectiveness of GnRH agonists in an adenomyosis population was observed in our Assisted Reproductive Technology center; i.e., a decline in serum level of cancer antigen 125 (to less than $35 \mathrm{U} / \mathrm{mL}$ ) before the commencement of ET is commonly observed among $\mathrm{GnRH}$ agonists-treated adenomyosis cases for at least 3 months. An increased endometrial receptivity with the use of $\mathrm{GnRH}$ agonists has been demonstrated in a study of mice with induced adenomyosis in terms of uterine expression of implantation markers (including homeobox A10, homeobox A11, integrin b3, and leukemia inhibitory factor) compared to non-treated mice [16].

Considering the wide variations in adenomyosis extent and severity and the corresponding diagnostic criteria, it is uncertain whether the beneficial effect of atosiban use depends on the clinical appearance of adenomyosis. The present study thus stratified the analyses by adenomyosis features and found an insignificant increase in pregnancy rates after atosiban therapy among patients with more extensive adenomyosis conditions. Future research with a large number of adenomyosis cases with diverse features is warranted to corroborate the findings of this study.

\section{Study limitations}

This was a retrospective study and thus confounding from various patient underlying characteristics may have affected our results. However, because there was no significant difference in baseline clinical and treatment characteristics between our study groups (see Table 1), this concern might be minimized. Second, the repeated pregnancy measures following several consecutive ET cycles for a given person were likely to be correlated. We thus applied advanced statistics (i.e., the GEE model) that carefully adjusted for withinperson dependency to ensure the validity of our results. Third, the generalizability of our results should be considered with caution because the study patients were from one center in Taiwan. Fourth, the relatively limited number of patients in each subgroup (e.g., few cases in the patient subgroups stratified by ultrasound features of adenomyosis; Table 3) might have affected the statistical power for detecting the therapeutic benefits of atosiban use.

In conclusion, our results show poorer pregnancy outcomes following ET among endometriosis patients with coexisting adenomyosis compared to those with endometriosis only or tubal infertility factor only. More extensive adenomyosis-affected endometriosis patients might benefit from atosiban use. Our results indicate that future research is urgently needed to identify effective and promising clinical strategies for enhancing the pregnancy outcomes of IVF treatment among infertile women suffering from adenomyosis.

\section{Declaration}


Permission for the study was obtained from the Institutional Review Board (IRB) of National Cheng Kung University Hospital (NCKUH) (A-ER-109-265). Since all study procedures were routine care and practice, the informed consents to study patients were waived by the IRB of NCKUH.

\section{Consent for publication}

Not applicable.

\section{Availability of data and materials}

The datasets generated and/or analysed during the current study are not publicly available as they contained individual patient information but are available from the corresponding author on reasonable request

\section{Competing interests}

The authors declare that they have no competing interests.

\section{Funding}

This work was supported by the Ministry of Science and Technology, Taiwan, under grant MOST 109-2320-B-006-047-MY3 (recipient: Huang-tz $\mathrm{Ou}$ ). The sponsor had no role in the design and conduct of the study; collection, management, analysis, and interpretation of the data; preparation, review, or approval of the manuscript; and decision to submit the manuscript for publication.

\section{Authors' contributions}

WMH and LCW acquire the data. OHT, WMH, and LCW contribute to the design of the study, and drafting of the article. OHT is the major contributor in writing the manuscript. All authors contributed to the analysis and interpretation of data, read and approved the final manuscript.

\section{Acknowledgments}

The authors wish to thank the staff members of the Assisted Reproductive Technology Center at National Cheng Kung University Hospital for their assistance with data collection.

\section{Data availability}

the datasets generated during and/or analyzed during the current study are available from the corresponding author on reasonable request.

\section{References}

1. Guo SW. The Pathogenesis of Adenomyosis vis-a-vis Endometriosis. J Clin Med. 2020;9(2):485.

2. Munro MG. Uterine polyps, adenomyosis, leiomyomas, and endometrial receptivity. Fertil Steril. 2019;111(4):629-40.

3. Fanchin R, Righini C, Olivennes F, Taylor S, de Ziegler D, Frydman R. Uterine contractions at the time of embryo transfer alter pregnancy rates after in-vitro fertilization. Human Reproduction. 1998;13(7):1968-74.

4. Pierzynski P, Reinheimer TM, Kuczynski W. Oxytocin antagonists may improve infertility treatment. Fertility and Sterility. 2007;88(1):213.e19-.e22.

5. Chou PY, Wu MH, Pan HA, Hung KH, Chang FM. Use of an oxytocin antagonist in in vitro fertilization-embryo transfer for women with repeated implantation failure: a retrospective study. Taiwan J Obstet Gynecol. 2011;50(2):136-40.

6. Lan VT, Khang VN, Nhu GH, Tuong HM. Atosiban improves implantation and pregnancy rates in patients with repeated implantation failure. Reprod Biomed Online. 2012;25(3):254-60.

7. Ng EH, Li RH, Chen L, Lan VT, Tuong HM, Quan S. A randomized double blind comparison of atosiban in patients undergoing IVF treatment. Hum Reprod. 2014;29(12):2687-94. 
8. He Y, Wu H, He X, Xing Q, Zhou P, Cao Y, et al. Administration of atosiban in patients with endometriosis undergoing frozenthawed embryo transfer: a prospective, randomized study. Fertil Steril. 2016;106(2):416-22.

9. Van den Bosch T, de Bruijn AM, de Leeuw RA, Dueholm M, Exacoustos C, Valentin L, et al. Sonographic classification and reporting system for diagnosing adenomyosis. Ultrasound Obstet Gynecol. 2019;53(5):576-82.

10. Mavrelos D, Holland TK, O'Donovan O, Khalil M, Ploumpidis G, Jurkovic D, et al. The impact of adenomyosis on the outcome of IVF-embryo transfer. Reprod Biomed Online. 2017;35(5):549-54.

11. Lessey BA, Kim JJ. Endometrial receptivity in the eutopic endometrium of women with endometriosis: it is affected, and let me show you why. Fertil Steril. 2017;108(1):19-27.

12. Huang QY, Rong $M H$, Lan $A H$, Lin $X M$, Lin XG, He RQ, et al. The impact of atosiban on pregnancy outcomes in women undergoing in vitro fertilization-embryo transfer: A meta-analysis. PLoS One. 2017;12(4):e0175501.

13. Liang YL, Kuo TC, Hung KH, Chen TH, Wu MH. Oxytocin antagonist for repeated implantation failure and delay of delivery. Taiwan J Obstet Gynecol. 2009;48(3):314-6.

14. Wu MH, Lin CW, Su PF, Lai EC, Sie FC, Mau YL, et al. Atosiban and pregnancy outcomes following in vitro fertilization treatment for infertile women requiring one, two, or more embryo transfer cycles: a longitudinal cohort study. Reprod Sci. 2020; 27(3):853-9.

15. Niu ZH, Chen Q, Sun YJ, Feng Y. Long-term pituitary downregulation before frozen embryo transfer could improve pregnancy outcomes in women with adenomyosis. Gynecol Endocrinol. 2013;29(12):1026-30.

16. Guo S, Li Z, Yan L, Sun Y, Feng Y. GnRH agonist improves pregnancy outcome in mice with induced adenomyosis by restoring endometrial receptivity. Drug Des Devel Ther. 2018;12:1621-31.

\section{Tables}

Table 1. Study participants' characteristics: overall and stratified by subgroup 


\begin{tabular}{|c|c|c|c|c|c|c|c|c|c|c|}
\hline \multirow[b]{2}{*}{ Characteristics } & \multicolumn{2}{|c|}{$\begin{array}{l}\text { Overall } \\
\text { (103 } \\
\text { patients, } 176 \\
\text { ET cycles) }\end{array}$} & \multicolumn{2}{|c|}{$\begin{array}{l}\text { Endometriosis } \\
\text { only } \\
\text { (34 patients, } \\
54 \text { ET cycles) }\end{array}$} & \multicolumn{2}{|c|}{$\begin{array}{l}\text { Endometriosis } \\
\mathbf{+} \\
\text { adenomyosis } \\
\text { (34 patients, } \\
66 \text { ET cycles) }\end{array}$} & \multicolumn{2}{|c|}{$\begin{array}{l}\text { Tubal only } \\
\text { (38 } \\
\text { patients, } 56 \\
\text { ET cycles) }\end{array}$} & \multirow{2}{*}{$\begin{array}{l}\text { Difference: endometriosis } \\
\text { only vs. endometriosis + } \\
\text { adenomyosis } \\
\text { p-value }\end{array}$} & \multirow{2}{*}{$\begin{array}{l}\text { Difference: } \\
\text { tubal only vs. } \\
\text { endometriosis } \\
+ \\
\text { adenomyosis } \\
\text { p-value }\end{array}$} \\
\hline & $\mathrm{N}$ & $\begin{array}{l}\text { Mean } \\
(\mathrm{sd}) \\
\text { or \% }\end{array}$ & $\mathrm{N}$ & $\begin{array}{l}\text { Mean } \\
\text { (sd) or } \\
\%\end{array}$ & $N$ & $\begin{array}{l}\text { Mean } \\
\text { (sd) or } \\
\%\end{array}$ & $\mathrm{~N}$ & $\begin{array}{l}\text { Mean } \\
(\mathrm{sd}) \\
\text { or \% }\end{array}$ & & \\
\hline $\begin{array}{l}\text { Maternal age } \\
\text { at ET (years) }\end{array}$ & 175 & $\begin{array}{l}35.92 \\
(4.64)\end{array}$ & 54 & $\begin{array}{l}36.56 \\
(4.57)\end{array}$ & 65 & $\begin{array}{l}36.03 \\
(4.69)\end{array}$ & 56 & $\begin{array}{l}35.18 \\
(4.62)\end{array}$ & 0.540 & 0.318 \\
\hline BMI & 176 & $\begin{array}{l}22.39 \\
(3.61)\end{array}$ & 54 & $\begin{array}{l}21.95 \\
(2.31)\end{array}$ & 66 & $\begin{array}{l}22.96 \\
(4.44)\end{array}$ & 56 & $\begin{array}{l}22.13 \\
(3.52)\end{array}$ & 0.134 & 0.264 \\
\hline $\mathrm{AMH}(\mathrm{ng} / \mathrm{mL})$ & 119 & $\begin{array}{l}2.17 \\
(1.60)\end{array}$ & 47 & $\begin{array}{l}2.19 \\
(1.54)\end{array}$ & 46 & $\begin{array}{l}2.02 \\
(1.25)\end{array}$ & 26 & $\begin{array}{l}2.37 \\
(2.21)\end{array}$ & 0.566 & 0.401 \\
\hline $\begin{array}{l}\text { Number of } \\
\text { embryos } \\
\text { transferred per } \\
\text { ET cycle }\end{array}$ & 176 & $\begin{array}{l}2.36 \\
(0.93)\end{array}$ & 54 & $\begin{array}{l}2.28 \\
(0.94)\end{array}$ & 66 & $\begin{array}{l}2.42 \\
(0.98)\end{array}$ & 56 & $\begin{array}{l}2.38 \\
(0.89)\end{array}$ & 0.408 & 0.773 \\
\hline Day of ET & & & & & & & & & 0.298 & 0.807 \\
\hline Day 1-3 & 107 & $61.8 \%$ & 28 & $52.8 \%$ & 41 & $64.1 \%$ & 38 & $67.9 \%$ & & \\
\hline Day 4-6 & 66 & $38.2 \%$ & 25 & $47.2 \%$ & 23 & $35.9 \%$ & 18 & $32.1 \%$ & & \\
\hline $\begin{array}{l}\text { ET failure } \\
\text { history }\end{array}$ & & & & & & & & & 0.168 & 0.257 \\
\hline None & 47 & $37.3 \%$ & 20 & $43.5 \%$ & 13 & $27.7 \%$ & 14 & $42.4 \%$ & & \\
\hline At least one & 79 & $62.7 \%$ & 26 & $56.5 \%$ & 34 & $72.3 \%$ & 19 & $57.6 \%$ & & \\
\hline \multicolumn{11}{|l|}{$\begin{array}{l}\text { Adenomyosis } \\
\text { subtype }\end{array}$} \\
\hline $\begin{array}{l}\text { Differentiation } \\
=\text { focal }\end{array}$ & 27 & $40.9 \%$ & & & 27 & $40.9 \%$ & & & & \\
\hline $\begin{array}{l}\text { Differentiation } \\
=\text { diffuse }\end{array}$ & 39 & $59.1 \%$ & & & 39 & $59.1 \%$ & & & & \\
\hline $\begin{array}{l}\text { Layer = } \\
\text { inner }\end{array}$ & 48 & $72.7 \%$ & & & 48 & $72.7 \%$ & & & & \\
\hline $\begin{array}{l}\quad \text { Layer = } \\
\text { middle or } \\
\text { outer }\end{array}$ & 18 & $27.3 \%$ & & & 18 & $27.3 \%$ & & & & \\
\hline $\begin{array}{l}\text { Location = } \\
\text { anterior wall }\end{array}$ & 5 & $7.6 \%$ & & & 5 & $7.6 \%$ & & & & \\
\hline $\begin{array}{c}\text { Location = } \\
\text { posterior wall }\end{array}$ & 61 & $92.4 \%$ & & & 61 & $92.4 \%$ & & & & \\
\hline Extent $<25 \%$ & 21 & $31.8 \%$ & & & 21 & $31.8 \%$ & & & & \\
\hline $\begin{array}{l}\text { Extent }=25- \\
50 \%\end{array}$ & 30 & $45.5 \%$ & & & 30 & $45.5 \%$ & & & & \\
\hline Extent $>50 \%$ & 15 & $22.7 \%$ & & & 15 & $22.7 \%$ & & & & \\
\hline $\begin{array}{l}\text { Type of } \\
\text { embryo } \\
\text { transferred }\end{array}$ & & & & & & & & & 0.648 & 0.257 \\
\hline Fresh & 74 & $42.5 \%$ & 21 & $39.5 \%$ & 22 & $33.8 \%$ & 31 & $55.4 \%$ & & \\
\hline
\end{tabular}




\begin{tabular}{|c|c|c|c|c|c|c|c|c|c|c|}
\hline $\begin{array}{l}\text { Frozen- } \\
\text { thawed }\end{array}$ & 100 & $57.5 \%$ & 32 & $60.4 \%$ & 43 & $66.2 \%$ & 25 & $44.6 \%$ & & \\
\hline $\begin{array}{l}\text { Atosiban } \\
\text { administration }\end{array}$ & & & & & & & & & 0.450 & 0.210 \\
\hline Yes & 79 & $45.4 \%$ & 30 & $55.6 \%$ & 30 & $46.9 \%$ & 19 & $33.9 \%$ & & \\
\hline No & 95 & $54.6 \%$ & 24 & $44.4 \%$ & 34 & $53.1 \%$ & 37 & $66.1 \%$ & & \\
\hline \multicolumn{11}{|l|}{$\begin{array}{l}\text { Pregnancy } \\
\text { outcomes }\end{array}$} \\
\hline $\begin{array}{l}\text { Biochemical } \\
\text { pregnancy } \\
\text { (yes) }\end{array}$ & 68 & $39.5 \%$ & 26 & $50.0 \%$ & 19 & $29.7 \%$ & 23 & $41.1 \%$ & $0.041^{\star}$ & 0.266 \\
\hline $\begin{array}{l}\text { Ongoing } \\
\text { pregnancy } \\
\text { (yes) }\end{array}$ & 51 & $29.3 \%$ & 19 & $35.8 \%$ & 15 & $22.7 \%$ & 17 & $30.9 \%$ & 0.170 & 0.418 \\
\hline Live birth (yes) & 44 & $25.4 \%$ & 19 & $35.2 \%$ & 11 & $16.9 \%$ & 14 & $25.9 \%$ & $0.038^{*}$ & 0.330 \\
\hline
\end{tabular}

Abbreviations: sd: standard deviation, BMI: body mass index, AMH: anti-Müllerian hormone, ET: embryo transfer.

Notes:

Difference in patient characteristics was tested by $t$-tests (for continuous variables) and chi-square tests (for dichotomous and categorical variables).

* indicates $p$-value $<0.05$.

Table 2. Results of IVF pregnancy outcomes between subgroups using generalized equation model analyses where repeated ET cycles for a given person were adjusted

\begin{tabular}{|c|c|c|c|c|c|c|}
\hline \multirow[b]{2}{*}{ Subgroup comparison } & \multicolumn{2}{|c|}{$\begin{array}{l}\text { Biochemical pregnancy } \\
\text { (patients: 103, ETs: 170) }\end{array}$} & \multicolumn{2}{|c|}{$\begin{array}{l}\text { Ongoing pregnancy } \\
\text { (>10 weeks) } \\
\text { (patients: 102, ETs: } \\
172 \text { ) }\end{array}$} & \multicolumn{2}{|c|}{$\begin{array}{l}\text { Live birth (>24 weeks) } \\
\text { (patients: 101, ETs: 171) }\end{array}$} \\
\hline & OR $(95 \% \mathrm{Cl})$ & $p$-value & OR $(95 \% \mathrm{Cl})$ & $\begin{array}{l}p- \\
\text { value }\end{array}$ & OR $(95 \% \mathrm{Cl})$ & $\begin{array}{l}p- \\
\text { value }\end{array}$ \\
\hline $\begin{array}{l}\text { Endometriosis only versus endometriosis + } \\
\text { adenomyosis (ref.) }\end{array}$ & $\begin{array}{l}2.981(1.307 \\
6.803)\end{array}$ & $0.009 * *$ & $\begin{array}{l}2.030(0.908 \\
4.541)\end{array}$ & 0.085 & $\begin{array}{l}2.694(1.151 \\
6.304)\end{array}$ & $0.022^{\star}$ \\
\hline $\begin{array}{l}\text { Tubal only versus endometriosis + } \\
\text { adenomyosis (ref.) }\end{array}$ & $\begin{array}{l}2.372(0.996 \\
5.651)\end{array}$ & 0.051 & $\begin{array}{l}1.737(0.719 \\
4.192)\end{array}$ & 0.220 & $\begin{array}{l}0.1 .797 \\
(0.694,4.655)\end{array}$ & 0.228 \\
\hline
\end{tabular}

Abbreviations: OR: odds ratio, Cl: confidence interval, ET: embryo transfer.

Note:

* indicates $p$-value $<0.05$ and ** indicates $p$-value $<0.001$.

Table 3. Results of IVF pregnancy with atosiban use or non-use among adenomyosis-affected women stratified by clinical ultrasound presentation 


\begin{tabular}{|llll|}
\hline \multicolumn{1}{|c|}{ Pregnancy outcomes } & Biochemical pregnancy & Ongoing pregnancy (>10 weeks) & Live birth (>24 weeks) \\
\hline Subtype & With vs. without atosiban (\%) & With vs. without atosiban (\%) & With vs. without atosiban (\%) \\
\hline Differentiation & & & \\
\hline Focal $(n=17)$ & $16.7 \%$ vs. $20.0 \%$ & $27 \%$ vs. $20.0 \%$ & $8.3 \%$ vs. $13.3 \%$ \\
\hline Diffuse $(n=21)$ & $35.3 \%$ vs. $33.3 \%$ & & $22.2 \%$ vs. $22.2 \%$ \\
\hline Layer & & $36.4 \%$ vs. $40.0 \%$ & $36.4 \%$ vs. $40.0 \%$ \\
\hline Inner $(n=10)$ & $36.4 \%$ vs. $40.0 \%$ & $10.5 \%$ vs. $20.7 \%$ & $5.3 \%$ vs. $14.3 \%$ \\
\hline Middle or outer $(n=28)$ & $22.2 \%$ vs. $25.0 \%$ & & $0.0 \%$ vs. $25.0 \%$ \\
\hline Location & & $0.0 \%$ vs. $25.5 \%$ & $17.2 \%$ vs. $17.2 \%$ \\
\hline Anterior wall $(n=3)$ & $0.0 \%$ vs $33.3 \%$ & $20.7 \%$ vs. $23.3 \%$ & $12.5 \%$ vs. $15.4 \%$ \\
\hline Posterior wall $(n=17)$ & $28.6 \%$ vs $26.7 \%$ & & $14.3 \%$ vs. $23.1 \%$ \\
\hline Extent & & $12.5 \%$ vs. $23.1 \%$ & $25.0 \%$ vs. $14.3 \%$ \\
\hline$\leq 25 \%(n=13)$ & $12.5 \%$ vs $23.1 \%$ & $14.3 \%$ vs $.28 .6 \%$ & \\
\hline $25-50 \%(n=16)$ & $28.6 \%$ vs $28.6 \%$ & $37.5 \%$ vs. $14.3 \%$ & \\
\hline$\geq 50 \%(n=10)$ & $42.9 \%$ vs $33.3 \%$ & & \\
\hline
\end{tabular}

Abbreviation: IVF: in vitro fertilization treatment.

Notes:

- $\mathrm{n}$ refers to the number of embryo transfers.

- Difference in pregnancy rate between atosiban-treated and non-treated patients in each subgroup was further tested using simple Fisher's exact tests (which were applied under consideration of low number of repeated embryo transfer cycles for a given person); there was no statistically significant difference between atosiban use and non-use in each group.

\section{Figures}


Figure 1: Forest plots for association of atosiban with IVF pregnancy outcome determined using generalized estimating equation analysis

Biochemical pregnancy

OR $\quad 95 \% \mathrm{CI}$

Endometriosis-only
group
Endometriosis+adenomyosis
group
Tubal-only
group

Favor no atosiban

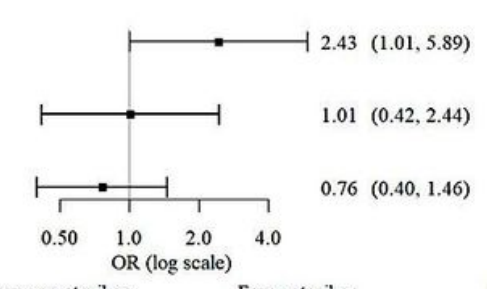

Favor atosiban
Ongoing pregnancy

OR $95 \% \mathrm{CI}$

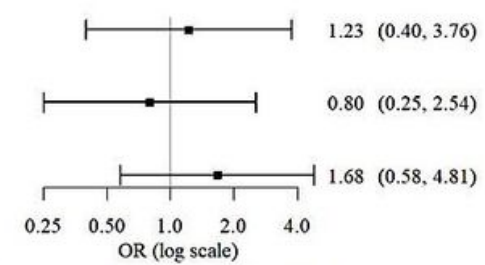

Favor no atosiban

Favor atosiban
Live birth

OR $95 \% \mathrm{CI}$

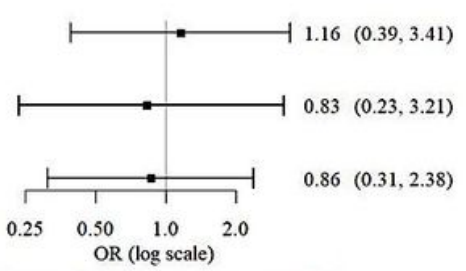

Favor no atosiban

Favor atosiban

Abbreviations: IVF: in vitro fertilization, OR: odds ratio, CI: confidence interval.

\section{Figure 1}

See image above for figure legend. 